\title{
Observing human interaction with physical devices
}

\author{
Cristina Massen
}

Received: 12 March 2009 / Accepted: 29 July 2009 / Published online: 18 August 2009

(C) The Author(s) 2009. This article is published with open access at Springerlink.com

\begin{abstract}
Previous study has shown that if we observe another person operating a tool or physical device, then the action rule of the observed action is automatically activated and can subsequently facilitate own actions. In this study, the mechanisms responsible for this automatic priming of actions are investigated. In two experiments, the question is raised whether priming arises from the observation of the physical device and its movements, or whether it is modulated by aspects of the person's behaviour. Whereas experiment 1 shows that priming effects are not influenced by the effector used by the observed person, experiment 2 demonstrates that they are modulated by the handle (and associated action rule) that is used to operate the device. These results suggest that motor resonance mechanisms are sensitive to the specific interaction between movements of an actor and associated movements of a physical device.
\end{abstract}

Keywords Motor resonance - Action priming Action observation · Man-machine interaction · Tool use . Imitation

\section{Observing human interaction with physical devices}

In recent years, evidence has accumulated that observing another person perform an action automatically activates corresponding motor programmes in the observer. Evidence

C. Massen ( $\square)$

Max-Planck-Institute for Human Cognitive and Brain Sciences, Leibniz Research Centre for Working Environment

and Human Factors, Ardeystrasse 67,

44139 Dortmund, Germany

e-mail:massen@ifado.de for this phenomenon of motor resonance has been obtained both in behavioural studies (e.g., Brass et al. 2001; Edwards et al. 2003; Dijkerman and Smit 2007) and in studies using neurophysiological, electrophysiological or neuroimaging methodology (e.g. Gallese et al. 1996; Rizzolatti et al. 2001; Fadiga et al. 1995; Iacoboni et al. 1999; Grafton et al. 1996). In many of the studies cited, the effects of observing simple transitive or intransitive movements ${ }^{1}$ were studied. However, the human action repertoire is by no means restricted to simple movements such as reaching for an object or pointing to it. In everyday life, we quite often interact with complex physical devices that serve as tools to achieve our goals. For example, we ride a bike or steer a car to reach other places faster and with less bodily effort. We row a boat when travelling on water. In the household, many different mechanical and electrical devices like washing machines, sewing machines, can or bottle openers are used to improve the effectiveness of our actions. In all of the examples cited, movements of the acting person are transformed into movements and/or effects of the physical device at hand. For example, when a person sitting in a rowboat wants the boat to go right (in the direction of motion), he or she moves his or her right arm and the oar handle towards the body, which causes the blade of the oar to move through the water in the opposite direction. Alternatively, he or she can also move the left arm and left oar handle away from the body, which causes the blade of the oar to move through the water in a direction towards the body. In this example, movements of the left or right arm are transformed into movements of the blades of the oars and finally into movements of the boat.

\footnotetext{
${ }^{1}$ Transitive movements involve manipulation of objects, whereas intransitive movements express ideas or feelings.
} 
This study is concerned with the effects of observing another person interacting with a physical device. From the perspective of simulation theory (Jeannerod 2001), the question arises whether observing the movements of the acting person automatically primes corresponding motor activity in the same way as with simpler actions without any devices involved. However, if action simulation subserves the function of understanding (Gallese et al. 1996; see also Rizzolatti et al. 1996), or predicting the outcome (cf. Schubotz and von Cramon 2002; Wilson and Knoblich 2005; Flanagan and Johansson 2003; Prinz 2006) of others' actions, then it might not be sufficient to just simulate the movements of the acting person. Instead, some kind of simulation of the behaviour of the physical device seems necessary to fully understand the actions and their environmental consequences. It might even be the case that humans neglect the movements of the acting person under these circumstances, and instead focus on the behaviour of the physical device instead. Three pieces of evidence from the literature on action observation and motor resonance might be taken as hints to suggest that participants could focus on the behaviour of the physical device when observing humans interacting with physical devices or machines.

First, evidence from single-cell recordings shows that activity of monkey F5 mirror neurons often relates to the function or meaning of an action, irrespective of the exact movement observed. For instance, Kohler et al. (2002) found mirror neurons that discharged both when the animal performed an action and when it only heard the actionrelated sound. Ferrari et al. (2005) described a type of toolresponding mirror neurons whose response was specific for the kind of tool used to perform an action.

Second, it has been argued, that the same system that is involved in simulating others' actions is also used for visual imagery and the prediction of behaviour of non-living objects or entities (cf. Grush 2004). For instance, Schubotz and von Cramon (2002) have demonstrated in a fMRI study that predicting perceptual events activates corresponding motor schemes in lateral premotor cortex. More specifically, it was shown that the prediction of the size of a stimulus activated premotor areas involved in hand movements, whereas the prediction of pitch in auditory sequential events engaged premotor areas involved in articulation. Other evidence suggests that the mirror neuron system responds to both human and robotic actions (Press et al. 2005, 2007; Gazzola et al. 2007, but see Kilner et al. 2003). Although there are also studies from the literature showing that cognitive processing of biological motion may differ from the processing of non-biological motion (e.g., Shiffrar and Freyd 1993; Press et al. 2005; Kilner et al. 2003), this evidence strongly suggests that motor resonance phenomena are by no means restricted to living human or non-human beings. Furthermore, action priming studies have revealed that the mere observation of stationary objects and tools may lead to an automatic activation of corresponding actions (e.g., Tucker and Ellis 1998, 2004; Grafton et al. 1997; Murata et al. 1997).

A third line of evidence, that might be relevant in our context, stems from the field of observational skill learning. Here, it has generally been shown that watching another person perform a motor skill (e.g., practising Tai Chi exercises or ballet postures) can have beneficial effects on one's own performance. In many studies on this topic, the observed movements were associated with some kind of external stimuli or object movements (e.g., a ski simulator, see Whiting et al. 1987), lending the opportunity for observational learning on the basis of the observed object movements (cf. Vogt 1995). In addition, Wulf et al. (2002, see also McNevin et al. 2003) have shown that, in motor skill acquisition in general, attention to more distal effects of one's own movements is more effective than attention to the movements themselves. On the basis of these results, it seems reasonable to predict motor resonance for movements of objects and physical devices when man-machine interaction is observed.

This study tries to shed light on the processing mechanisms that enable people to understand (and eventually imitate) observed interaction with physical devices. We used a paradigm in which participants have to operate a mechanical lever device (cf. Massen and Prinz 2007a, b). The lever is mounted horizontally on a plastic plate and movable around one of two possible pivotal points (see Fig. 1).

The task is to touch one of two targets with the lever by moving its handle in the appropriate direction (towards or away from the body), taking into account the momentarily active pivotal point of the lever. For example, if the lever is movable around the left pivotal point, as shown in Fig. 1, the handle on the right side of the lever has to be moved away from the body (i.e. arm extension is required) to touch the far target, and towards the body (arm flexion) to touch the near target. When the handle is on the right, the

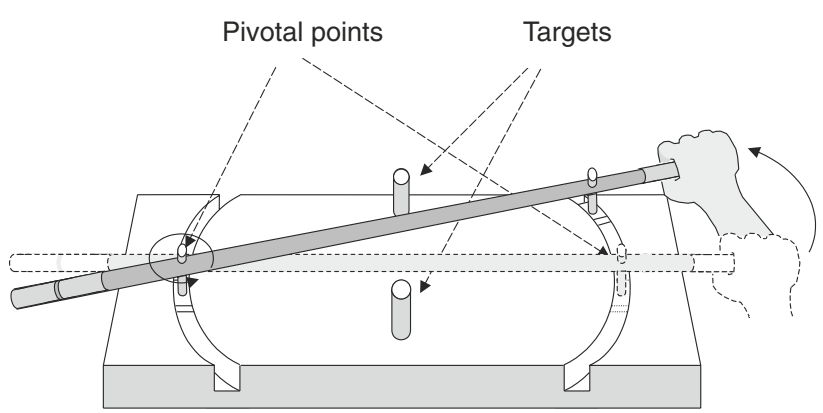

Fig. 1 Lever apparatus used in the experiments. From 'movements, actions and tool-use actions' by C. Massen and W. Prinz, in press, Philosophical Transactions of the Royal Society B. Copyright 2009 by The Royal Society 
left pivotal point thus implements a compatible target-tomovement mapping (or action rule) because bodily movement direction and direction of the target reached are always the same. If the lever is movable around the right pivotal point, then the handle has to be moved towards the body to touch the far target point, and away from the body to touch the near target point. Hence, when the handle is on the right, the right pivotal point implements an incompatible action rule, because the direction of the bodily movement and the direction of the target reached are always opposite to each other.

In the experimental session, two participants are sitting side by side, each operating their own lever device. Taking turns in acting, they have to observe the lever action of the other person in trial $n-1$ and subsequently execute a lever action in trial $n$. Trial transitions from $n-1$ to $n$ are manipulated in four conditions. In the first condition, the pivotal point (i.e. the action rule), the target and also the bodily movement direction (towards or away from the body) are repeated from trial $n-1$ (observation) to trial $n$ (execution). In the second condition, only the pivotal point is repeated, whereas target and bodily movement direction change. In the third condition, the pivotal point changes, the target is repeated, and the movement also changes. In the fourth condition, both the pivotal point and the target change, but the bodily movement direction is repeated.

With this setup, it is possible to analyse the effects of observing the other's action in trial $n-1$ on executing an action in trial $n$. A previous study on this topic (Massen and Prinz 2007b) has yielded the result that participants show better performance (in terms of reaction times and/or error rates) when they have observed a lever action with the same pivotal point (i.e. the same action rule) in the previous trial, even when target and bodily movement directions were different. These results suggest that the observation of complex actions leads to an automatic activation of the action rule observed. However, they leave open the question whether participants simulate the behaviour of the physical device observed (ignoring the movements of the person observed), or whether their simulation includes aspects of the person's behaviour.

In order to investigate this question, in this study, experimental conditions were created that differed with respect to the behaviour of the person observed, but were constant with respect to the functioning of the physical device. In experiment 1 , participants observed a model person acting either with the same or the other arm (but with the same handle), which did not affect observational priming. In experiment 2, participants observed a model that executed actions using the handle on the other side of the lever device, but with the same arm. In this group, the action rule implemented by each pivotal point reverses for the model (i.e., if the right handle is used, the left pivotal point implements the compatible rule and the right one the incompatible, but if the left handle is used, the left pivotal point implements the incompatible rule and the right one the compatible). The results show that priming effects are modulated by the way the lever is operated and suggest that action priming is sensitive to the specific interaction between human movements and associated movements of a physical device.

\section{Experiment 1}

In experiment 1 , a model and a participant were sitting side by side, taking turns in operating their lever devices. The participants always used their right arm and the right handle of their lever device. One group of participants observed a model who executed lever actions with the left arm (but using the right handle, see Fig. 2), the other group of participants observed a model who used the right arm and the right handle, similar to the participants did. If participants simulate the lever device, then the effects of observation (i.e. better performance in the conditions where the pivotal point/action rule is repeated) should not be modulated by the effector used by the model person. However, if group differences are found, then it may be concluded that participants include specific aspects of the model's behaviour (i.e. the effector used) in their simulation.

\section{Method}

Participants

Sixty right-handed participants from the subject pool of the Max-Planck-Institute in Leipzig served as participants, 30 in each observation condition. They were paid 7 Euros for their participation. The local ethics committee approved the study, and all the participants gave informed consent.

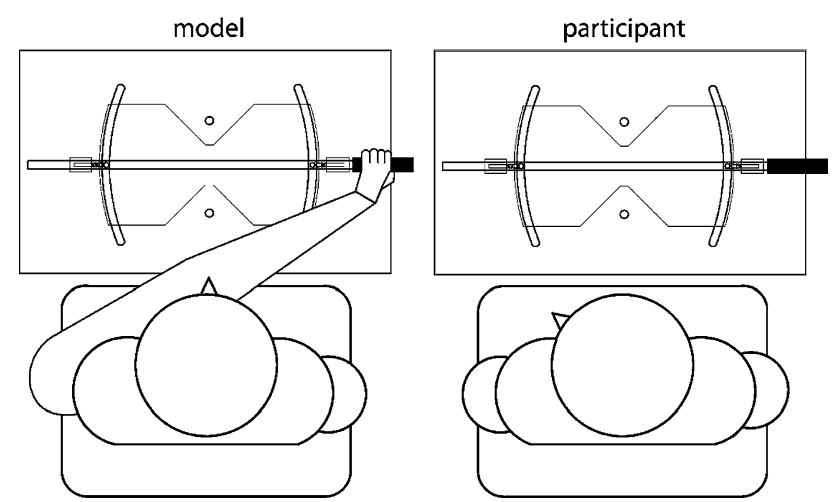

Fig. 2 Experimental setup in experiment 1. The figure shows the group who observed a model using the left arm to operate the lever device 


\section{Apparatus}

The apparatus consisted of a metallic lever that was mounted horizontally on a plastic plate $(62.5 \mathrm{~cm} \times 44.5 \mathrm{~cm})$ and movable around two possible pivotal points (see Fig. 1). These were located in a distance of $32.5 \mathrm{~cm}$ to each other and in a distance of $11 \mathrm{~cm}$ from the near end of the respective lever handle. Each pivotal point could be locked and unlocked by a magnet attached beneath it. If one of them was unlocked, the lever was movable around the other one; if both were locked, the lever was not movable at all. Two possible target points (wired, elastic pins with a light diode on top of it), which were situated orthogonal to the lever (each at a distance of $10 \mathrm{~cm}$ from the lever and of $16 \mathrm{~cm}$ from the pivotal points), could be touched with the lever by moving its handle in the appropriate direction (towards versus away from the body in the horizontal plane), depending on the momentarily 'active' pivotal point. A reversal movement (initiation of the movement in the wrong direction and subsequent correction) was possible.

If one of the target pins was touched by the lever (participants were instructed to stop at the target, but it was difficult to go through the target position anyway because of the wired elastic pins), then the light diode on top of it was turned on. Light diodes on each pivotal point signalled whether it was active at the moment (if the light diode was turned on, then the lever was movable around this point). The light diodes could be turned on and off by signals via the parallel port of a standard IBM-compatible computer, and the pivotal points could be locked or unlocked by it. In turn, the computer received signals about the position of the lever: Whether it was in the resting position, left it in the direction away from the body or left it in the direction towards the body, and touched the far-target point or touched the near-target point.

\section{Design and procedure}

The experiment took place in a dimly illuminated room. Model persons ${ }^{2}$ were already familiar with the task and were counterbalanced across groups. In order to motivate new participants to pay attention to the expert's actions, they were instructed to take turns in acting with an expert (model) person and to pay attention to this expert's actions. The model person was instructed to also watch the actions of the new participant in turn. The model person, who was sitting on the left, was always the one to begin, acting in trials with odd numbers. The participant performed in trials

\footnotetext{
${ }^{2}$ The reason for using an expert in the experiments was that we excluded all those trials from analysis in which the model made an error and we wanted to minimize the number of these errors.
}

with even numbers. Only the data of the participant were analysed.

Both lever devices were connected to two different parallel ports of the same computer. This allowed us to independently control trial events for the two lever devices. For each participant, there were four different combinations of pivotal (left/right) and target points (far/near). As trial events for both participants were independent from one another, this resulted in 16 possible types of transitions between observation trials in $n-1$ and execution trials in $n$. These can be reduced to four relevant transition conditions (pivot and target repeated; pivot repeated/target changed; pivot changed/target repeated, pivot changed/target changed). All the possible transitions appeared equally often and in a randomized order. We also controlled for the effects of trial $n-2$. Participants were informed about the task, which was to touch one of the target points with the lever by moving the lever's handle in the appropriate direction. Each trial started with a preparation interval of $1600 \mathrm{~ms}$. Then one of the target point diodes and one of the pivotal point diodes were turned on to inform the subject about the active pivotal point and the target point to touch. Simultaneously with the appearance of the instruction lights, the other pivotal point was unlocked to allow the lever to rotate around the active, lighted pivotal point. The acting subject had to decide in which direction to move the lever handle. Reaction time was defined as the time from the lighting of the diodes to the lever's leaving of the resting position. As soon as the participant reacted and the lever left its resting position, all the diodes were turned off until one of the target points was reached and touched by the lever, which caused the light diode on the target point to again light up. Movement time was measured from the point in time when the lever left its resting position until one of the target points was touched.

After one of the target points had been touched, the lever was to be moved back to the resting position, all the lights were turned off and both pivotal points were locked. Then, the next trial started.

There was a practice block of 20 trials (except for the number of trials, the practice block was identical to the experimental blocks of the respective condition). Participants went through one experimental block of 256 trials. Thus, they observed 128 trials executed by the model and executed 128 trials themselves. The experiment took approximately $25 \mathrm{~min}$.

\section{Results}

Dependent variables were reaction times for correct responses and error rates. Errors were all reactions that were initiated in the wrong movement direction, irrespective 
Table 1 Reaction Times (RTs, in ms) and Percentage Error (PE) in experiments 1 and 2 as a function of group and transition condition

\begin{tabular}{|c|c|c|c|c|c|c|c|c|c|}
\hline \multirow[b]{3}{*}{ Group } & & \multicolumn{8}{|c|}{ Transition condition } \\
\hline & & \multicolumn{2}{|c|}{$\begin{array}{l}\text { Pivot repeated/ } \\
\text { target repeated }\end{array}$} & \multicolumn{2}{|c|}{$\begin{array}{l}\text { Pivot repeated/ } \\
\text { target changed }\end{array}$} & \multicolumn{2}{|c|}{$\begin{array}{l}\text { Pivot changed/ } \\
\text { target repeated }\end{array}$} & \multicolumn{2}{|c|}{$\begin{array}{l}\text { Pivot changed/ } \\
\text { target changed }\end{array}$} \\
\hline & & RT & $\mathrm{PE}$ & RT & PE & RT & PE & $\mathrm{RT}$ & $\mathrm{PE}$ \\
\hline \multirow[t]{2}{*}{ Exp 1: Effector } & Same & 420 & 23.4 & 426 & 26.2 & 431 & 28.3 & 435 & 28.0 \\
\hline & Different & 452 & 18.9 & 459 & 20.5 & 471 & 24.6 & 465 & 24.6 \\
\hline \multirow[t]{2}{*}{ Exp 2: Handle } & Same & 409 & 23.1 & 424 & 24.3 & 431 & 29.6 & 426 & 30.8 \\
\hline & Different & 437 & 21.4 & 430 & 23.6 & 432 & 23.6 & 433 & 25.3 \\
\hline
\end{tabular}

Fig. 3 Reaction Times (in ms) and Error Rates (in \%) as a function of pivot transition and group in experiment 1. Error bars show standard deviations
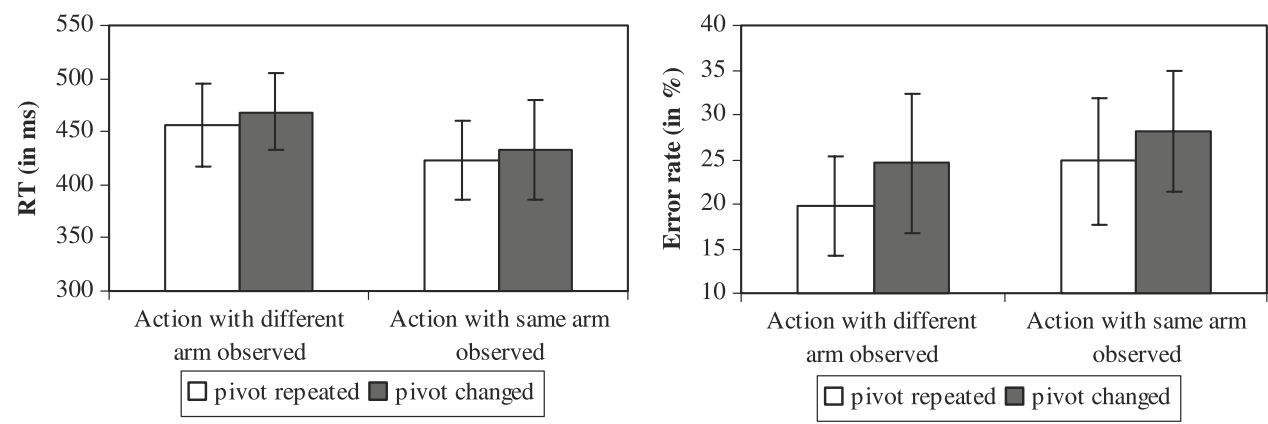

of whether they were corrected or not. All the trials after an error made by the model in trial $n-1$ were excluded from analysis. ${ }^{3}$ Anticipations $(\mathrm{RT}<100 \mathrm{~ms})$ were also excluded from analysis. As there were no effects on movement times, they are not reported in the following. An alpha level of 0.05 was used for all the statistical tests.

\section{Reaction times}

Median reaction times for correct responses were calculated for each transition condition and each participant. Data were analysed with a three-way ANOVA with group (same effector observed versus different effector observed) as between-subjects factor and pivot transition (pivot repeated versus pivot changed), and target transition (target repeated versus target changed) as within-subjects factors. Mean RTs are depicted in Table 1.

The ANOVA revealed a significant main effect of pivot transition $\left(F_{(1,58)}=8.6 ; P<0.01 ; \mathrm{MSE}=874.4\right)$, which was due to faster reaction times when participants had observed an action with the same pivot/rule in the previous trial (cf. Fig. 3, left panel). Neither the main effect of group $\left(F_{(1,58)}=2.8 ; P=0.10 ; \mathrm{MSE}=25119.2\right)$ nor any other

\footnotetext{
${ }^{3}$ Performance of the model participants in the trials preceding those that were analysed for participants did not differ significantly when analysed as a function of group and transition condition of the subsequent trial. In experiment 1 , there was a trend towards slower RTs and MTs when models used the left arm, but this trend was not significant. In experiment 2, there were no effects of group or subsequent transition condition for model participants.
}

effect reached statistical significance (all $F_{\mathrm{S}}<1$ ). Analyses within groups indicated that the main effect of pivot transition was significant in the group who observed a model using the right $\operatorname{arm}\left(F_{(1,29)}=5.2 ; P<0.05 ; \mathrm{MSE}=586.4\right)$ and just missed statistical significance in the group who observed a model using the left arm $\left(F_{(1,29)}=3.9\right.$; $P=0.057, \mathrm{MSE}=1162.5)$.

\section{Error rates}

For each participant, error rates in each of the four transition conditions were calculated. The means are depicted in Table 1. The ANOVA revealed a significant main effect of pivot transition $\left(F_{(1,58)}=13.9 ; P<0.01 ; \mathrm{MSE}=72.5\right)$ that was because of participants committing fewer errors when they had observed an action with the same pivot in the previous trial (cf. Fig. 3, right panel). Analyses within groups showed that the main effect of pivot transition was significant in the group who observed a model using the right arm $\left(F_{(1,29)}=5.4 ; \mathrm{MSE}=62.1 ; P<0.05\right)$ and also in the other group $\left(F_{(1,29)}=8.5 ; \mathrm{MSE}=82.9 ; P<0.01\right)$. Neither the main effect of group $\left(F_{(1,58)}=1.6 ; \mathrm{MSE}=677.3 ; P=0.20\right)$ nor any other effect reached statistical significance (all $F$ s $<1)$.

\section{Discussion}

Experiment 1 was conducted to investigate whether the effects of observing a model person operating a physical 
device are modulated by the effector used by the model. The results show that participants in both groups made fewer errors and were faster when they had observed an action with the same pivotal point and action rule in the previous trial. However, these effects were not influenced by the effector used by the model. One further aspect of the results of experiment 1 should be noted, though. Although the main effect of group did not reach statistical significance, participants who observed a model using the same arm were faster than participants who observed a model using the other arm. Note however, that they also made more errors than participants of the other group (although again not significant), suggesting a speed-accuracy tradeoff here. If this pattern is not due to a priori differences between the two groups, it could indicate that the observation of a model's behaviour biases the participant towards not only faster, but also more error-prone response when the model's behaviour and response requirements closely match those of his own.

One possible explanation for the lack of a clear influence of the observed effector on priming effects is that the participants simply imagine the behaviour of the physical device. In this context, it should be noted that the observation effects we report are not due to perceptual priming arising from the task cues. In our previous study, the effects were still found under conditions where the task cues of the model are either not visible for the observer, or not present because the pivot remains constant within a person. Hence, priming arising from observation must be due to the observation of the movements of the lever device according to the explanation offered above.

Another possibility is that changing the effector used by the model might be too weak a manipulation to test the hypothesis that priming effects are influenced by the movements of the person observed. Although effector-specific action priming effects have been reported in the literature (e.g. Bach et al. 2007; Buccino et al. 2001; Gillmeister et al. 2008), there are also studies on intentional imitation suggesting that humans often neglect the effector with which an observed action is executed (e.g., Bekkering et al. 2000; Wohlschläger et al. 2003). Therefore, in experiment 2 , the way how the model operated the lever device was changed in a more substantial way to test whether the results of experiment 1 would be replicated under these conditions.

\section{Experiment 2}

In experiment 2, one half of the participants observed a model operating the lever device using the left handle (cf. Fig. 4), whereas the other half observed a model that used

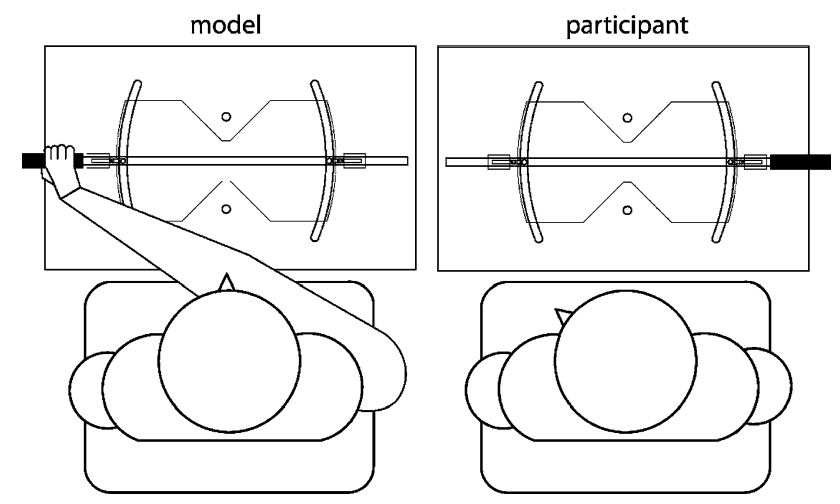

Fig. 4 Experimental setup in experiment 2. The figure shows the group, who observed a model using the left handle to operate the lever device

the right handle to operate it. If the left handle is used, then the action rule implemented by each pivotal point reverses: The left pivotal point defines an incompatible and the right one a compatible rule, as compared to a compatible rule defined by the left pivotal point and an incompatible rule defined by the right pivotal point if the right handle is used. Thus, the action rules that have to be applied to touch one of the targets reverse, when the left handle is used. Note however, that the movements of the physical device remain exactly the same, regardless of whether the right or left handle is used to operate it.

If participants only simulate the physical device and its movements, then they should always benefit from a repetition of the pivotal point across trials, even if this means that an action with a different action rule is observed when the model uses the left handle. Conversely, if the participants only simulate the abstract action rule applied by the model, then priming effects should reverse when the model used the left handle. In this case, performance should be better when the pivotal point changes because then the action rule remains the same. If participants take into account both aspects of the action, then priming effects should be reduced or even disappear when the model uses the left handle.

\section{Method}

Participants

Seventy-two right-handed participants from the subject pool of the Max-Planck Institute in Leipzig served as participants. None of them had participated in experiment 1 . They were paid 7 Euros for their participation. The local ethics committee approved the study, and all the participants gave informed consent. 
Apparatus

The apparatus was the same as in experiment 1 .

Design and procedure

Participants used the right arm and the right handle to operate their lever device. They were randomly assigned to one of two groups in which model persons used their right arm to operate either the left handle (first group) or the right handle (second group). Model persons as well as sitting positions of participants were counterbalanced across groups. In all other respects, the procedure was the same as in experiment 1 .

There was a practice block of 20 trials. Participants went through one experimental block of 256 trials. The experiment took approximately $25 \mathrm{~min}$.

\section{Results}

\section{Reaction times}

Data were analysed with a three-way ANOVA with handle group (model uses the same handle versus model uses the other handle) as between-subjects factor and pivot transition (pivot repeated versus pivot changed) and target transition (target repeated versus target changed) as within-subjects factors. Mean RTs are depicted in Table 1. The ANOVA yielded no significant main effect of pivot transition $\left(F_{(1,70)}=3.2\right.$; MSE $=670.1 ; P=0.08)$, but a significant interaction of pivot transition and handle group $\left(F_{(1,70)}=5.0 ; \mathrm{MSE}=\right.$ 670.1, $P<0.05$ ). This interaction (cf. Fig. 5, left panel) was due to the fact that participants who observed actions with the same handle had faster reaction times when the pivot of the device was the same between trials $\left(F_{(1,35)}=7.5\right.$; MSE $=730.6 ; P<0.01)$, whereas there was no effect of pivot transition for the other group $\left(F_{(1,35)}<1\right.$; MSE $\left.=609.6\right)$. Furthermore, the ANOVA revealed no main effect of target transition $\left(F_{(1,70)}<1 ; \operatorname{MSE}=697.1\right)$ nor of the interaction between target transition and handle group
$\left(F_{(1,70)}=1.8 ; \mathrm{MSE}=697.1\right)$. The only other significant effect was a three-way interaction of target transition, pivot transition and handle group $\left(F_{(1,70)}=5.5 ; \quad P<0.05\right.$; $\mathrm{MSE}=617.5)$. Analyses within groups indicated that for participants who observed a model using the same handle, there was a significant interaction between pivot transition and target transition $\left(F_{(1,35)}=6.2 ; P<0.05 ; \mathrm{MSE}=570.2\right)$, indicating that the main effect of pivot transition was mainly driven by fast RTs in the condition where both, pivot and target, were repeated. No such effect occurred in participants who observed a model using the other handle $\left(F_{(1,35)}<1 ; \operatorname{MSE}=664.8\right)$.

\section{Error rates}

Error rates are depicted in Table 1. The ANOVA yielded a significant main effect of pivot transition, which was due to lower error rates when participants had observed a lever action with the same pivot in the previous trial $\left(F_{(1,70)}=13.1 ; \mathrm{MSE}=98.8 ; P<0.01\right)$. The interaction of pivot transition and handle group just missed statistical significance $\left(F_{(1,70)}=3.9 ; \mathrm{MSE}=98.8 ; P=0.054\right)$. Analyses within groups indicated that the main effect of pivot transition was significant for participants who observed a model using the same handle $\left(F_{(1,35)}=12.0 ; \mathrm{MSE}=128.3\right.$; $P<0.01)$, whereas it was not significant for the other group $\left(F_{(1,35)}=2.0 ;\right.$ MSE $\left.=69.3\right)$. There were no significant effects of handle group $\left(F_{(1,70)}=1.1 ; \mathrm{MSE}=811.0\right)$, target transition $\left(F_{(1,70)}=3.0 ; \mathrm{MSE}=60.7\right)$ or any other effect (all $F$ s $<1$ ). Figure 5 (right panel) shows error rates for both groups, collapsed across the conditions with pivot repeated versus pivot not repeated.

\section{Discussion}

The results of experiment 2 show that participants do not simply simulate the physical device and its movements. Although exactly the same lever movements were observed in the two observation conditions of experiment 2, priming through observation was only evident for
Fig. 5 Reaction Times (in ms) and Error Rates (in \%) as a function of pivot transition and group in experiment 2 . Error bars show standard deviations
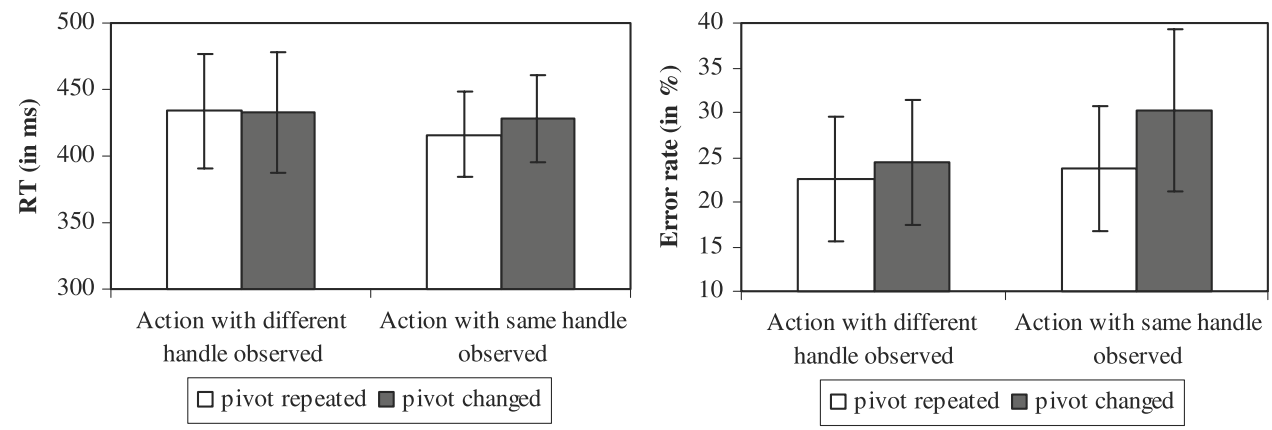
participants who observed a model operating the same handle of the lever.

Furthermore, the results are also not consistent with the hypothesis that participants only simulate the action rule applied by the model, ignoring the functioning of the tool. In this case, priming effects should have reversed for those participants who observed actions with the other handle. This was not the case, however. The pattern of results obtained suggests that priming effects are sensitive to both the action rules applied by the model and the behaviour of the tool. ${ }^{4}$

A somewhat surprising feature of the results is the fact that for RTs, the effect of pivot repetition was modulated by the target factor for participants who observed a model using the same handle. This result seems to suggest that priming is dependent on both pivot and target repetitions and shows that target information is also taken into account. However, as there were no effects of target transition (or of its interaction with pivot transition) either in our previous study or in experiment 1 of this study, caution seems warranted in placing too much emphasis on this aspect of the results. Further study is required to clarify whether this is a reliable result, and if so, under what conditions it emerges.

It might be objected that the observation of a model using the other handle to operate the lever device goes along with the observation of a somewhat awkward grip, because models used their right arm at the left handle. In another experiment from our lab, which has not been included in this study due to space limitations, models used the left arm at the left handle and the results were essentially the same, with no priming emerging from observation. This renders any explanation in terms of awkwardness of the grip observed unlikely.

\section{General discussion}

The experiments conducted in this study aimed at further investigating priming mechanisms in the observation of others interacting with a physical device. More specifically, the question was raised whether participants would focus on the behaviour of the physical device, or whether observational effects would reflect the behaviour of the human model. Whereas experiment 1 showed that priming effects are not modulated by the effector that is used by the model,

\footnotetext{
${ }^{4}$ This interpretation is further corroborated by an analysis of the data of the model participants as a function of handle condition and transition condition in the previous trial. They showed significant priming effects for repeating the pivot of the lever device even when they observed actions with the other handle. However, these priming effects were significantly smaller than those obtained when actions with the same handle were observed.
}

experiment 2 demonstrated that they are sensitive to the handle and action rule used by the model.

On the one hand, these results replicate previous results (cf. Massen and Prinz 2007b) in demonstrating that participants show better performance when they have observed a lever action with the same pivotal point and action rule in the previous trial. On the other hand, the results obtained extend those of our previous study in showing that participants neither exclusively focus on the behaviour of the physical device, nor exclusively simulate the abstract action rule applied by the model. Instead, the pattern of results suggests that action priming effects are rather specific and reflect the combination of both aspects of the action.

There are many studies from the literature on imitation that suggest that participants decompose an observed action into hierarchically organized subcomponents, of which the goal component is the most important and receives more attention than the means with which it is achieved (e.g., Bekkering et al. 2000; Wohlschläger et al. 2003). However, other studies (e.g., Bird et al. 2007) have failed to find evidence for such an imitation-specific dominance of goals in action observation. Instead, they have shown that the saliency of an action component in the respective action context determines what is activated in action observation. Consistent with these results, for the more complex actions investigated here, the abstract way (the how) an action is performed seems to be more important than the target of the action.

On the one hand, the way how the action is performed is determined by the pivotal point or functioning of the mechanical device. Therefore, one might expect participants to focus attention on its behaviour and disregard the behaviour of the model (e.g., whether it uses the right or left handle as a means to make the lever move). More generally speaking, if humans operate technical devices, they themselves can be considered a means to make the device behave in the desired way, and their behaviour might therefore be neglected in action observation.

On the other hand, there is also evidence for a special role of human and/or biological motion for action observation and action simulation. For instance, Kilner et al. (2003) demonstrated that observing another human making incongruent movements had a significant interference effect on simultaneously executed movements, whereas no interference occurred when participants observed a robotic arm making incongruent movements. Press et al. (2005) reported that observation of an incongruent robotic movement can lead to interference with a simultaneously executed action, but to a lesser degree than observation of an incongruent human movement. These results suggest a special preference for simulating movements of a person as opposed to simulating those of objects or physical devices. 
Our data suggest that participants take into account both, the movements of the actor as well as those of the physical device, when observing human interaction with physical devices. A recent study on cooperative action, although tackling a different research question, is in accordance with this view. Shibata et al. (2007) used near-infrared spectroscopy to measure brain activation in participants, who watched movie clips of a person passing an object to a receiver. The movies differed with respect to the appropriateness of the receiving action for the specific passing action displayed. For instance, an inappropriate action would be to try to catch a big object that approaches straight from the front using a precision grip, instead of a power grip. The results showed greater activation for receiving actions that were appropriate for the specific passing action in brain areas typically associated with the mirror neuron system. Hence, motor activation reflected not only the receiving action, but also the prior passing action. The authors conclude that the mirror neuron system might be involved in the understanding of cooperative action. In a similar way as the understanding of cooperative action between humans, the understanding of human interaction with physical devices depends on both, the action of the human actor and the behaviour of the physical device. The actor's movements have to be selected according to the mechanical structure of the physical device to generate the desired environmental effect. The present results are in accordance with the results by Shibata et al. (2007) in showing that motor resonance depends on both, the movements of an actor and the associated behaviour of the physical device. The fact that priming effects were only sensitive to those aspects of the human movement, which are of functional relevance for the action, suggests that a function of this kind of motor resonance could be needed to facilitate action understanding or action prediction.

Acknowledgments I would like to thank Henrik Grunert for constructing the lever devices and Katharina Horstkotte, Babett Wedau, Sascha Weis, Ramona Kaiser and Julia Michael for their help with data acquisition.

Open Access This article is distributed under the terms of the Creative Commons Attribution Noncommercial License which permits any noncommercial use, distribution, and reproduction in any medium, provided the original author(s) and source are credited.

\section{References}

Bach P, Peatfield NA, Tipper SP (2007) Focusing on body sites: the role of spatial attention in action perception. Exp Brain Res 178:509-517

Bekkering H, Wohlschläger A, Gattis M (2000) Imitation of gestures in children is goal-directed. Q J Exp Psychol 53:153-164
Bird G, Brindley R, Leighton J, Heyes C (2007) General processes, rather than "goals", explain imitation errors. J Exp Psychol Hum Percept Perform 33:1158-1169

Brass M, Bekkering H, Prinz W (2001) Movement observation affects movement execution in a simple response task. Acta Psychol 106:3-22

Buccino G, Binkofski F, Fink GR, Fadiga L, Fogassi L, Gallese V, Seitz RJ, Zilles K, Rizzolatti G, Freund HJ (2001) Action observation activates premotor and parietal areas in a somatotopic manner: an fMRI study. Eur J Neurosci 13:400-404

Dijkerman HC, Smit MC (2007) Interference of grasping observation during prehension, a behavioural study. Exp Brain Res 176:387-396

Edwards MG, Humphreys GW, Castiello U (2003) Motor facilitation following action observation: a behavioural study in prehensile action. Brain Cogn 53:495-502

Fadiga L, Fogassi L, Pavesi G, Rizzolatti G (1995) Motor facilitation during action observation: a magnetic stimulation study. J Neurophysiol 73:2608-2611

Ferrari PF, Rozzi S, Fogassi L (2005) Mirror neurons responding to observation of actions made with tools in monkey ventral premotor cortex. J Cogn Neurosci 17:212-226

Flanagan JR, Johansson RS (2003) Action plans used in action observation. Nature 424:769-771

Gallese V, Fadiga L, Fogassi L, Rizzolatti G (1996) Action recognition in the premotor cortex. Brain 119:593-609

Gazzola V, Rizzolatti G, Wicker B, Keysers C (2007) The anthropomorphic brain: the mirror neuron system responds to human and robotic actions. Neuroimage 35:1674-1684

Gillmeister H, Catmur C, Liepelt R, Brass M, Heyes C (2008) Experience-based priming of body parts: a study of action imitation. Brain Res 1217:157-170

Grafton ST, Arbib MA, Fadiga L, Rizzolatti G (1996) Localization of grasp representations in humans by PET: 2 observation compared with imagination. Exp Brain Res 112:103-111

Grafton ST, Fadiga L, Arbib MA, Rizzolatti G (1997) Premotor cortex activation during observation and naming of familiar tools. Neuroimage 6:231-236

Grush R (2004) The emulation theory of representation: motor control, imagery, and perception. Behav Brain Sci 27:377-442

Iacoboni M, Woods RP, Brass M, Bekkering H, Mazziotta JC, Rizzolatti G (1999) Cortical mechanisms of human imitation. Science 286:2526-2528

Jeannerod M (2001) Neural simulation of action: a unifying mechanism for motor cognition. Neuroimage 14:103-109

Kilner JM, Paulignan Y, Blakemore SJ (2003) An interference effect of observed biological movement on action. Curr Biol 13:522-525

Kohler E, Keysers C, Umiltà MA, Fogassi L, Gallese V, Rizzolatti G (2002) Hearing sounds, understanding actions: action representation in mirror neurons. Science 297:846-848

Massen C, Prinz W (2007a) Programming tool-use actions. J Exp Psychol Hum Percept Perform 33:692-704

Massen C, Prinz W (2007b) Activation of action rules in action observation. J Exp Psychol Learn Mem Cogn 33:1118-1130

McNevin NH, Shea CH, Wulf G (2003) Increasing the distance of an external focus of attention enhances learning. Psychol Res 67:22-29

Murata A, Fadiga L, Fogassi L, Gallese V, Raos V, Rizzolatti G (1997) Object representation in the ventral premotor cortex (area F5) of the monkey. J Neurophysiol 78:2226-2230

Press C, Bird G, Flach R, Heyes C (2005) Robotic movement elicits automatic imitation. Cogn Brain Res 25:632-640

Press C, Gillmeister H, Heyes C (2007) Sensorimotor experience enhances automatic imitation of robotic action. Proc R Soc Lond B Biol Sci 274:2509-2514

Prinz W (2006) What re-enactment earns us. Cortex 42:515-517 
Rizzolatti G, Fadiga L, Gallese V, Fogassi L (1996) Premotor cortex and the recognition of motor actions. Cogn Brain Res 3:131-141

Rizzolatti G, Fogassi L, Gallese V (2001) Neurophysiological mechanisms underlying the understanding and imitation of action. Nat Rev Neurosci 2:661-670

Schubotz RI, von Cramon DY (2002) Predicting perceptual events activates corresponding motor schemes in lateral premotor cortex: an fMRI study. Neuroimage 15:787-796

Shibata H, Suzuki M, Gyoba J (2007) Cortical activity during the recognition of cooperative actions. Neuroreport 18:697-701

Shiffrar M, Freyd JF (1993) Timing and apparent motion path choice with human body photographs. Psychol Sci 4:379-384

Tucker M, Ellis R (1998) On the relations between seen objects and components of potential actions. J Exp Psychol Hum Percept Perform 24:830-846
Tucker M, Ellis R (2004) Action priming by briefly presented objects. Acta Psychol 116:185-203

Vogt S (1995) On the relations between perceiving, imagining and performing in the learning of cyclical movement sequences. Br J Psychol 86:191-216

Whiting HTA, Bijlard ML, den Brinker BP (1987) The effect of the availability of a dynamic model on the acquisition of a complex cyclical action. Q J Exp Psychol 39:43-59

Wilson M, Knoblich G (2005) The case for motor involvement in perceiving conspecifics. Psychol Bull 131:460-473

Wohlschläger A, Gattis M, Bekkering H (2003) Action generation and action perception in imitation: An instance of the ideomotor principle. Philos Trans R Soc Lond B Biol Sci 358:501-515

Wulf G, McConnel N, Gartner M, Schwarz A (2002) Enhancing the learning of sport skills through external-focus feedback. J Mot Behav 34:171-182 